\title{
Emerging Therapies for Bipolar Depression
}

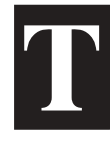

his ACADEMIC HIGHLIGHTS section of The Journal of Clinical Psychiatry presents the highlights of the planning teleconference "Emerging Therapies for Bipolar Depression," which was held February 9, 2006. This report was prepared by the CME Institute of Physicians Postgraduate Press, Inc. and was supported by an educational grant from Cyberonics, Inc.

The teleconference was chaired by Lauren B. Marangell, M.D., Mood Disorders Center, Department of Psychiatry, Baylor College of Medicine, Houston, Tex. The faculty were David J. Kupfer, M.D., Western Psychiatric Institute and Clinic, University of Pittsburgh School of Medicine, Pittsburgh, Pa.; Gary S. Sachs, M.D., Department of Psychiatry, Harvard Medical School, Massachusetts General Hospital, and Partners Bipolar Treatment Center, Boston; and Alan C. Swann, M.D., Department of Psychiatry and Behavioral Sciences, University of Texas Medical School, Houston.

Faculty disclosure: In the spirit of full disclosure and in compliance with all ACCME Essential Areas and Policies, the faculty for this CME article were asked to complete a statement regarding all relevant financial relationships between themselves or their spouse/partner and any commercial interest (i.e., any proprietary entity producing health care goods or services consumed by, or used on, patients) occurring within at least 12 months prior to joining this activity. The CME Institute has resolved any conflicts of interest that were identified. The disclosures are as follows: Dr. Marangell is a consultant for Cyberonics, Eli Lilly, GlaxoSmithKline, Medtronic, and Pfizer; has received grant/research support from Bristol-Myers Squibb, Cyberonics, Eli Lilly, and Neuronetics; and has received honoraria from and is a member of the speakers or advisory boards for Cyberonics, Eli Lilly, Forest, and GlaxoSmithKline. Dr. Kupfer is a consultant for Servier Amerique and is on the advisory boards of Pfizer, Forest, and SolvayWyeth. Dr. Sachs is a consultant for Abbott, GlaxoSmithKline, Janssen, Eli Lilly, Bristol-Myers Squibb, Novartis, Elan, Sanofi, Sigma-Tau, and AstraZeneca; has received grant support from Abbott and Janssen; and has received honoraria from Abbott, GlaxoSmithKline, Janssen, Eli Lilly, Bristol-Myers Squibb, Solvay, Novartis, Sanofi, AstraZeneca, and Pfizer. Dr. Swann has received grant/research support from Abbott, Pfizer, Janssen, CIBA, Eli Lilly, GlaxoSmithKline, Shire, Novartis, and UCB Pharma; is a consultant for Abbott, GlaxoSmithKline, Janssen, Shire, Novartis, Ortho-McNeil, and AstraZeneca; and is on the speaker's bureau for Abbott, Eli Lilly, Pfizer, GlaxoSmithKline, Parke-Davis, and Ortho-McNeil.

The opinions expressed herein are those of the faculty and do not necessarily reflect the views of the CME provider and publisher or the commercial supporter.

Dr. Marangell began by stating that bipolar depression is a serious and potentially lethal phase of bipolar disorder with marked rates of suicidality. The challenges of treating bipolar depression begin with the difficulty of making an accurate diagnosis and are complicated by rapid cycling and treatment resistance. Each of these issues will be discussed.

\section{Course of Illness and Illness Outcome in Bipolar Depression}

To put the symptoms and illness outcomes of bipolar depression in proper context, David J. Kupfer, M.D., began by reviewing the symptoms of the 4 domains of bipolar disorder: manic mood and behavior, dysphoric mood and behavior, psychotic symptoms, and cognitive symptoms. Bipolar disorder is a chronic psychiatric illness characterized by depression and at least 1 manic or hypomanic episode during the lifetime course of the illness. ${ }^{1}$ Patients with bipolar disorder can have symptoms in more than one of these domains concurrently.

\section{Diagnosis}

Dr. Kupfer emphasized that the most important issue of bipolar disorder is making the correct diagnosis at the outset. One study ${ }^{2}$ assessed the accuracy of diagnoses using selfadministered questionnaires. Data from the first 600 respondents showed that bipolar disorder was misdiagnosed $69 \%$ of the time. Those who were misdiagnosed received a mean of 3.5 other diagnoses and consulted 4 physicians before receiving an accurate diagnosis. The most common incorrect diagnosis was unipolar depression (60\%), followed by an anxiety disorder (26\%) and schizophrenia (18\%). Of the misdiagnosed patients, $35 \%$ were symptomatic for more than 10 years before the correct diagnosis was made. Similar findings were reported in a study ${ }^{3}$ of the underrecognition of bipolar disorder in patients with major depressive episodes. Of 250 patients, $72 \%$ were misdiagnosed with unipolar depression and the remaining $28 \%$ were correctly diagnosed with bipolar I or II disorder. Dr. Kupfer asserted that misdiagnosis is the most common feature of bipolar disorder.

Dr. Kupfer then explained that it is important to recognize and treat bipolar depression because it is about 3 times more pervasive than mania. ${ }^{4}$ The mean duration of depressive episodes in bipolar disorder is longer than that of manic episodes and becomes chronic in about $20 \%$ of patients. ${ }^{5}$ The long-term outcome of bipolar disorder includes repeated episodes. A prospective study ${ }^{6}$ of 146 patients with bipolar I disorder found that patients were symptom-free only about $50 \%$ of the time. About $32 \%$ of the time, they exhibited depressive symptoms, with much less time spent in manic episodes $(9 \%)$ or cycling or mixed episodes $(6 \%)$.

\section{Impact on Daily Living}

According to Dr. Kupfer, bipolar disorder has a substantial negative impact on daily living. ${ }^{1}$ Patients with mood disorders are more likely to report declines in job status and income, less likely to marry, and more likely to report deficits in psychosocial functioning compared with controls. ${ }^{7}$ Further, between $30 \%$ and $60 \%$ of individuals diagnosed with bipolar disorder fail to regain full function in terms of vocational and social performance. ${ }^{8}$ In a study to determine the psychosocial impact of bipolar dis- 
order in a U.S. community sample, Calabrese and colleagues ${ }^{1}$ found that subjects who screened positive on the Mood Disorder Questionnaire (MDQ) reported significantly $(\mathrm{p}<.0001)$ more difficulties with work-related performance, social/leisure activities, and social/family interactions compared with MDQ-negative subjects. Significantly $(\mathrm{p}<.0001)$ more MDQ-positive subjects than negative subjects were ever fired or laid off or had a supervisor who was unhappy with their work, behavior, or attitude. MDQ-positive women reported more disruption in social and family life, whereas a quarter of MDQ-positive men reported being jailed, arrested, and convicted of crimes other than drinking while driving.

\section{Morbidity and Mortality in Bipolar Depression}

Dr. Kupfer stated that both longevity and general medical health are severely compromised in individuals with bipolar disorder. Bipolar disorder is a leading cause of death by suicide, violence, and homicide (Figure 1). ${ }^{9}$ Suicidal ideation and suicide attempts are thought to be higher in bipolar depression than in any other psychiatric condition. Almost $80 \%$ of patients with bipolar depression have reported suicidal ideation, ${ }^{10}$ and nearly half of all patients with bipolar disorder have made at least 1 suicide attempt. ${ }^{11} \mathrm{Com}-$ pleted suicide occurs more frequently during the depressive episode than during any other period in the life of a bipolar patient. ${ }^{12}$ In addition, morbidity and mortality in bipolar disorder-and particularly bipolar depression-are associated with medical illnesses such as cardiovascular disease, cerebrovascular disease, and respiratory disease, as well as a considerable amount of psychiatric comorbidity and substance abuse (Table 1). ${ }^{13}$

Dr. Kupfer submitted that although a great deal has been written about psychiatric comorbidity as affecting the outcome of medical illness, little has been published about the reverse. Dr. Kupfer offered obesity as an example-are psychiatric outcomes

Figure 1. Standardized Mortality Ratios (95\% CI) for Patients With Bipolar Disorder in Sweden, 1973-1995

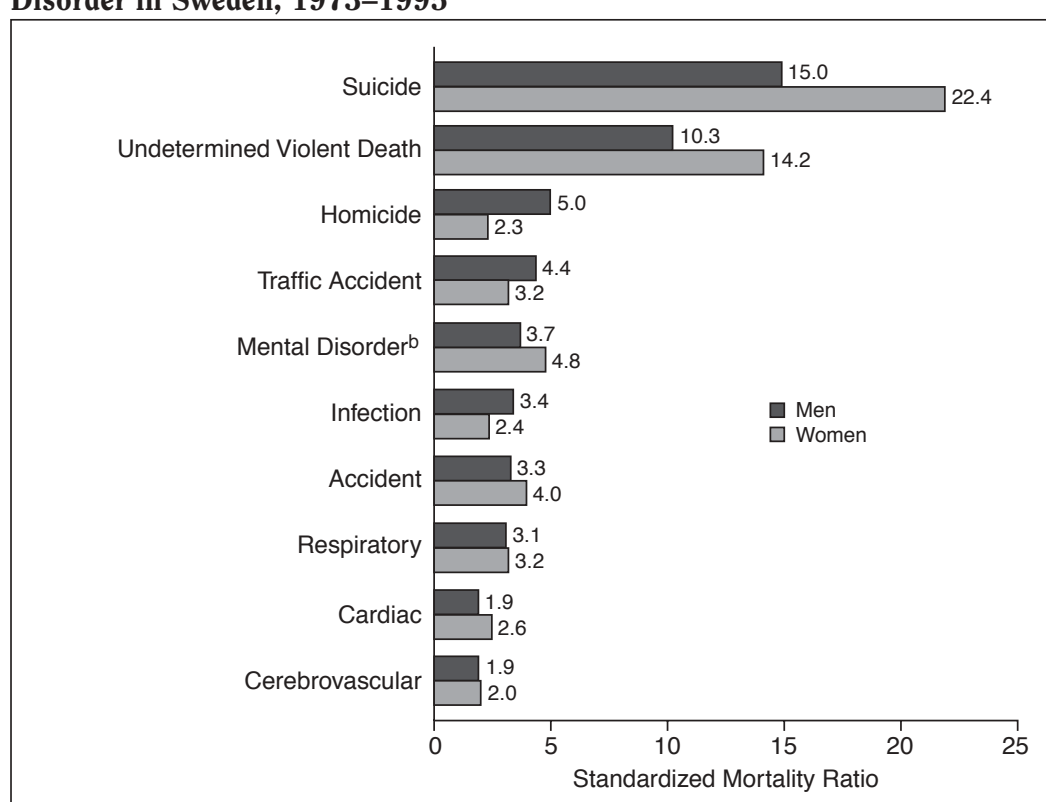

a Data from Osby et al. ${ }^{9}$ Standardized mortality ratio is calculated by dividing the observed number of deaths by the expected number of deaths.

${ }^{\mathrm{b}}$ Mental disorder as a cause of death in bipolar disorder includes dementia, psychosis, depression, alcohol addiction/abuse, drug addiction, and developmental disorders.

affected by obesity? A National Institute of Mental Health-supported longterm study ${ }^{14}$ examined 175 patients with bipolar I disorder who entered the study in the mid-1990s. Fagiolini and colleagues ${ }^{15}$ used these data to compare the time to depressive recurrence for obese versus nonobese patients with bipolar I disorder. Sixty-two patients were classified as obese and 113 were classified as nonobese. The data showed that over a longitudinal period of active treatment to prevent recurrence of depression, mania, or mixed episodes, obese patients had a much shorter time to a depressive recurrence than nonobese patients. Among the 46 obese patients who entered the preventative maintenance phase, 15 (32.6\%) experienced a depressive recurrence compared with $11(13.9 \%)$ of the 79 nonobese patients. From these data, Dr. Kupfer surmised that there might be a connection between obesity and the likelihood of having a new bipolar depressive episode.

A review ${ }^{16}$ of the available data indicates that the medical burden that

Table 1. Comorbidity of Bipolar
Disorder With Other Disorders ${ }^{\mathrm{a}}$
\begin{tabular}{|lc|}
\hline & Mean Rate of \\
Comorbid Condition & Comorbidity (\%) \\
\hline Anxiety disorder & 71 \\
Any Axis I disorder & 65 \\
Overweight & 58 \\
Substance use disorder & 56 \\
Alcohol abuse & 49 \\
Social phobia & 47 \\
Other drug abuse & 44 \\
Posttraumatic stress & 39 \\
disorder & \\
Personality disorder & 36 \\
Migraine & 28 \\
Obesity & 21 \\
Binge-eating disorder & 13 \\
Panic disorder & 11 \\
Obsessive-compulsive & 10 \\
disorder & \\
Type 2 diabetes & 10 \\
Hypothyroidism & 9 \\
\hline anata from Krishnan. & \\
\hline
\end{tabular}

exists in bipolar disorder is not solely related to the use of medications. Dr. Kupfer suggested that other contributors, such as the presence of a major psychiatric condition, the development of risk factors, an inability to change behavior, and even a certain amount of metabolic and genetic vulnerability 
to medical diseases, might be inherent in those individuals who are likely to develop bipolar disorder. The relationship between bipolar disorder and negative health behaviors as well as medical illness needs to be further clarified by additional studies.

\section{Conclusion}

Dr. Kupfer described the course of bipolar disorder as "hectic and variable — a 'roller coaster' — for both patients and clinicians."17(pg 593) He went on to say that hypomania can surge into mania and then plummet into months of major depression. Patients can also have a persistent subsyn- dromal minor depression that can move into euthymia.

Several different factors influence the course and outcome of bipolar depression. Both the immediate and longterm treatments for bipolar depression present several different possible outcomes, which make the impact of intervention in bipolar depression somewhat complex and difficult to assess. Dr. Kupfer concluded that treatments for bipolar disorder are not effective enough to always bring a patient out of an episode of major bipolar depression without the likelihood of moving very quickly either into a new manic episode or persistent subsyndromal depression.

\section{Treatment of Rapid-Cycling Bipolar Disorder}

Gary S. Sachs, M.D., defined rapid cycling as a condition in which patients have 4 or more manic or depressive episodes of at least 2 weeks' duration in a year. The term rapid cycling was first used by Dunner and Fieve ${ }^{18}$ in 1974 and was subsequently adopted by the Diagnostic and Statistical Manual of Mental Disorders, Fourth Edition $(\mathrm{DSM}-\mathrm{IV})^{19}$ as a course specifier for bipolar disorder. The DSM-IV, however, added that episodes are demarcated by either partial or full remission for at least 2 months or a switch to an episode of opposite polarity.

\section{Risks of Rapid Cycling}

Dr. Sachs compared 2 studies that have helped to define and clarify the risks of rapid cycling. The first ${ }^{20}$ used data from the first 500 participants in the Systematic Treatment Enhancement Program for Bipolar Disorder (STEP-BD). The other ${ }^{21}$ was an earlier study that used data from the Stanley Foundation International Mood Disorder Research Center in Sardinia, Italy.

Dr. Sachs stated that past estimates of gender differences in rapid-cycling bipolar disorder have indicated that more women than men are rapid cyclers. Because women tend to be overrepresented in clinical populations, however, the relationship is likely distorted. The STEP-BD ${ }^{20}$ and Sardinia ${ }^{21}$ data indicated a more modest difference in the proportion of women to men who met criteria for rapid cycling.

High rates of rapid cycling have long been associated with bipolar II disorder. Interestingly, rapid cycling was found among only $6 \%$ of participants with bipolar I disorder and $31 \%$ of participants with bipolar II disorder in the Sardinia data ${ }^{21}$ compared with $20 \%$ in both groups in STEP-BD. ${ }^{20}$ One explanation for this dissimilarity may be a difference in definition. For example, some bipolar II disorder in the Sardinia ${ }^{21}$ study may have been diagnosed as bipolar I disorder in STEP-BD. ${ }^{20}$

Next, Dr. Sachs described data from a 1-year prospective study ${ }^{22}$ of illness course in 539 outpatients with and without rapid cycling. A total of 206 (38.2\%) of the patients had rapid cycling, and $333(61.8 \%)$ did not have rapid cycling. Rapid cycling was more prevalent in patients with bipolar I disorder $(41.3 \%)$ than in patients with bipolar II disorder (27.9\%). The proportion of patients who had lifetime substance abuse was higher in patients with rapid cycling than in patients without rapid cycling $(45.4 \%$ and
$36.4 \%$, respectively), as was lifetime diagnosis of any anxiety disorder (50.2\% and $30.7 \%$, respectively). About half of the patients with rapid cycling had anxiety disorders compared with only one third of the patients without rapid cycling. About $40 \%$ of patients who were physically or sexually abused as children had rapid cycling compared with $24 \%$ of patients without rapid cycling. Parental psychiatric history also played a role. Patients with rapid cycling had parents with mood disorders and substance abuse at slightly higher rates than those without rapid cycling $(62.7 \%$ and $51.1 \%$, respectively, for mood disorders; $35.6 \%$ and $22.3 \%$, respectively, for parental substance abuse).

Dr. Sachs outlined the causes of affective mood switch and rapid cycling (Table 2). Sleep quality is one of the most objective measures of improvement or deterioration. ${ }^{23}$ Patients with bipolar disorder have a predisposition or vulnerability to perturbations in circadian rhythms and sleep-wake cycles; therefore, sleep quality and/or quantity is a key marker of impending relapse and an indication of treatment response. Dr. Sachs pointed out that genetics is a fairly new addition to the list of potential causes of rapid cycling. The serotonin promoter (5-HTTLPR) gene and the catechol-O-methyltransferase (COMT) gene have been associated with sensitivity to antidepressants. Long-form homozygotes have also been found to be associated with somewhat higher rates of relapses and may be associated with a greater vulnerability to rapid cycling. ${ }^{24}$ Green and colleagues ${ }^{25}$ recently reported that genetic factors may be important in influencing susceptibility to clinical subtypes of bipolar disorder. In that study, the valine allele at the Val66Met polymorphism of the brain-derived neurotrophic factor (BDNF) gene was associated with increased susceptibility to rapid-cycling bipolar disorder.

\section{Therapeutic Strategies}

Dr. Sachs next turned his attention to the challenges of treating rapid 


\begin{tabular}{l} 
Table 2. Potential Causes of Affective Mood Switch and Rapid Cycling \\
\begin{tabular}{|lc|}
\hline Affective Mood Switch & Rapid Cycling \\
\hline Sleep disturbance, loss, apnea & Neurologic factors \\
Alcohol/substance abuse & Brain injury/head trauma \\
Electroencephalogram abnormality & Mental retardation \\
Rapid discontinuation of lithium & Multiple sclerosis \\
Antidepressant use or discontinuation & Neuroendocrine factors \\
Interpersonal factors & Hypothyroidism \\
Conflict/trauma & Female sex \\
Grief & Psychotropic drugs \\
Success & Alcohol \\
Loss of support systems & Stimulants \\
Seasonality & Antidepressant drugs \\
East-west travel & Genetic risk factors \\
& BDNF (Val66Met) \\
& 5 -HTTLPR \\
& COMT 11 \\
\hline Abbreviations: 5-HTTLPR = serotonin transporter gene, BDNF = brain-derived neurotrophic factor, \\
COMT = catechol-O-methyltransferase gene.
\end{tabular} \\
\hline
\end{tabular}

cycling. Clinicians often confuse controlled trials that examine treatment specifically for rapid cycling with trials that show the impact of having a history of rapid cycling on the outcome of treatment for a single manic episode or a single depressive episode. Controlled trials for treating rapid cycling reveal a broader impact on the course of illness, but there is a rather surprising paucity of data.

Lithium. In 1974, Dunner and Fieve $^{18}$ reported that lithium prophylaxis failure may be related to the frequency of affective episodes. Lithium nonresponse was reported more often in patients who had the highest rate of rapid cycling. Relapse rates of rapid cyclers was double that of non-rapid cyclers ( $82 \%$ versus $41 \%$, respectively). Dr. Sachs stated that although a higher rate of relapse is often seen in patients who have more frequent cycles, Dunner and Fieve's findings do not represent a valid reason to preclude lithium for rapid cycling.

Antidepressants. Some evidence $^{26,27}$ indicates that the single most effective treatment of rapid cycling is to discontinue antidepressants. Dr. Sachs commented that although this idea is attractive, it is not based on clinical trial data per se. More systematic observation on the impact of antidepressants on rapid cycling over the next few years will shed more light on the feasibility of this recommendation.
Anticonvulsants. Only 2 trials with anticonvulsants have been reported. $\mathrm{One}^{28}$ was a trial of lamotrigine monotherapy, which was the first doubleblind, placebo-controlled, long-term maintenance evaluation of a large population of prospectively defined rapid-cycling patients. The other ${ }^{29}$ was a head-to-head trial that compared lithium with divalproex. Dr. Sachs presented relevant details from each study.

Lamotrigine versus placebo. The first study ${ }^{28}$ was a maintenance trial of patients with rapid-cycling bipolar disorder. Initially, lamotrigine was added to each patient's current psychotropic medication and titrated to clinical effect during an open-label phase of the study. Once the patients had stabilized, the other treatments were tapered, leaving the patients on lamotrigine monotherapy. The intent-to-treat cohort of 182 patients who stayed well for 2 weeks on lamotrigine treatment were randomly assigned to placebo $(\mathrm{N}=89)$ or lamotrigine $(\mathrm{N}=93)$ for the 6month double-blind maintenance phase. Five patients withdrew or were lost to follow-up; efficacy analysis included 177 patients (placebo $\mathrm{N}=87$; lamotrigine $\mathrm{N}=90$ ).

Overall, the percentage of patients who completed the 26-week randomized phase and were clinically stable on monotherapy without evidence of relapse was significantly $(\mathrm{p}<.05)$ greater in the lamotrigine group than in the placebo group (Figure 2) ${ }^{28}$ The

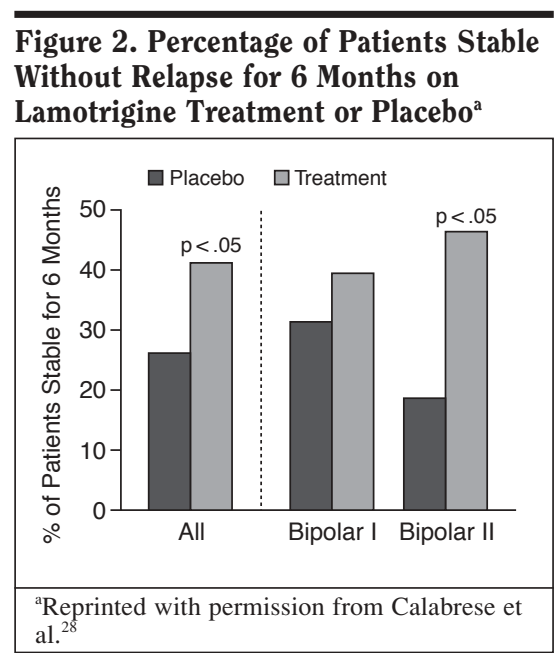

difference between the treatment group and placebo group was not statistically significant for the bipolar I subtype, but was significant ( $46 \%$ vs. $18 \%$, respectively; $\mathrm{p}=.04$ ) for the bipolar II subtype. Other outcomes for this dataset followed the same pattern. When overall survival in study was evaluated, the differences favored lamotrigine. Calabrese and colleagues ${ }^{28}$ concluded that lamotrigine may be a well-tolerated, effective mood stabilizer with prophylactic properties when used as monotherapy in some patients with rapid-cycling bipolar disorder and may be especially effective as a mood stabilizer in patients with bipolar II disorder.

Lithium versus divalproex. Next, Dr. Sachs described a more recent double-blind maintenance trial by Calabrese and colleagues ${ }^{29}$ that compared treatment with lithium to treatment with divalproex. The study enrolled 254 patients in an open-label acute stabilization phase. Eligible participants had to meet DSM-IV criteria for bipolar I or bipolar II disorder, have had rapid cycling during the 2 months prior to study entry, and have a history of at least 1 episode of hypomania, mania, or mixed state within 3 months of study entry. In this stabilization phase, patients were treated with the combination of lithium and divalproex. About three fourths (76\%) discontinued the study prematurely owing to poor adherence, nonresponse, and 
intolerable side effects. Sixty patients (24\%) entered the maintenance phase and were randomly assigned to treatment with lithium $(\mathrm{N}=32)$ or divalproex $(\mathrm{N}=28)$ monotherapy. The criteria for randomization were relatively liberal in that patients who had a recently high depression score on the Montgomery-Asberg Depression Rating Scale (MADRS) were included.

Dr. Sachs commented that none of the outcome comparisons were statistically significant and that only $16 \%$ of the patients who received lithium were able to complete the 20-month, doubleblind phase compared with $29 \%$ of the divalproex group. Fifty-six percent of the lithium group experienced a relapse versus $50 \%$ of the divalproex group. Surprisingly, the data showed more depressive relapse in the patients receiving lithium and more manic relapse in patients who received divalproex. Results for both drugs were disappointing.

Conclusions. Dr. Sachs summarized the conclusions that can be drawn from these 2 studies. ${ }^{28,29}$ Lamotrigine did not have any benefit over placebo for bipolar I patients in the first study, and little difference was noted between lithium and divalproex in the doubleblind phase of the second study; however, a rather modest response to the combination of divalproex and lithium in the open phase was reported.

Other strategies. Bauer and Whybrow $^{30}$ published a case report of a single patient with rapid-cycling affective illness who benefited from thyroid therapy. No controlled trials for treating rapid cycling with conventional or atypical antipsychotics have been reported.

\section{STEP-BD Rapid-Cycling Treatment Pathway}

Dr. Sachs outlined the following series of decision points that help guide clinicians in the appropriate treatment of rapid cycling.

- Determine the need for acutephase antidepressant treatment. Review the current symptom acuity and cycle frequency over the last 4 episodes. If the last 4 episodes of depression have been relatively brief-maybe even shorter than the time required for the onset of antidepressant action-an antidepressant is probably not indicated.

- Identify possible secondary factors for rapid cycling (see Table 2).

- Gradually taper any antidepressants (reduce dosage by $20 \%$ to $30 \%$ per month).

- Optimize anticycling agents. If the patient is taking an anticycling agent already, add another one (lithium or valproate). Consider lamotrigine for patients with bipolar II disorder.

- Evaluate outcome using systematic assessments and encourage patients to chart their mood after the patient has been off antidepressant treatment for at least 16 weeks.

- On the basis of assessment results, if the patient is recovering, continue the course of treatment. If not, add additional anticycling agent(s). Evaluate safety and patient tolerance. If the patient is not recovering, consider additional anticycling agents, ECT, or perhaps explorative agents. Review and select putative mood-stabilizing agents (for example, lithium and an atypical antipsychotic). Consider adding psychotherapy.

- Determine need to shift priority from anticycling to treatment of acute depression or mania.

Dr. Sachs advised that it is important to recognize that rapid cycling is a course specifier and that a period of rapid cycling is a marker for the propensity to relapse. Managing rapidcycling bipolar patients with add-on anticycling agents may help to diminish the role of antidepressant agents that might promote cycling.

\section{Long-Term Treatment of Bipolar Depression}

Alan C. Swann, M.D., stated that the first episode in bipolar disorder is typically depression, which then becomes the most dominant problem for most patients with bipolar disorder. ${ }^{31,32}$ Most psychosocial impairment and the vast majority of severe or completed suicide attempts occur during depressive episodes. Because depressive episodes in bipolar disorder are so common, Dr. Swann stated that the lack of effective treatments appears surprising. To date, only one treatment regimen, a combination of 2 medications, has gained approval from the U.S. Food and Drug Administration (FDA) for use in treating depressive episodes in bipolar disorder.

\section{Treatment Phases}

Dr. Swann explained that the 3 treatment phases for bipolar disorder are acute, continuation, and maintenance (Table 3). ${ }^{33}$ Each phase has specific goals, and one cannot move to the next phase until the treatment goals of the previous phase have been met. The goal of treating the index episode is safety and symptomatic recovery, which usually takes up to 2 months. Dr. Swann focused on the long-term treatment phases-continuation and maintenance.

Continuation phase. The goal of the continuation phase is a return to baseline functioning in a patient who has achieved symptomatic improvement. Patients should be able to live in their usual situation, and they should be able to return to the work they did prior to the episode. This phase is a high-risk period for suicide, relapse, and other complications, so another goal of the continuation phase is to address these concerns and reduce risk. Treatments that were effective in treating the index episode should be continued and adjusted for tolerability and practicality.

According to Dr. Swann, nonpharmacologic treatments may be instituted during this phase as well. Cognitive- 


\begin{tabular}{|c|c|c|c|}
\hline \multirow[b]{2}{*}{ Strategy } & \multicolumn{3}{|c|}{ Phase } \\
\hline & Acute & Continuation & Maintenance \\
\hline Goal & Syndromal recovery & Functional recovery & Stability \\
\hline Time & $0-8 \mathrm{wk}$ & $1-12 \mathrm{mo}$ & Indefinite \\
\hline \multicolumn{4}{|l|}{ Treatment } \\
\hline Pharmacologic & $\begin{array}{l}\text { Maximize mood stabilizers, } \\
\text { adjunctive treatments }\end{array}$ & $\begin{array}{l}\text { Adjust for tolerability, begin } \\
\text { taper of adjunctive treatments }\end{array}$ & $\begin{array}{l}\text { Optimize, address } \\
\text { prodromal symptoms }\end{array}$ \\
\hline Nonpharmacologic & $\begin{array}{l}\text { Structure, support, education } \\
\text { of family }\end{array}$ & $\begin{array}{l}\text { Behavioral treatments, systems } \\
\text { measures, institute monitoring }\end{array}$ & $\begin{array}{l}\text { Strategies to optimize adaptation, monitor for } \\
\text { prodromal symptoms and other psychiatric, } \\
\text { medical, or social changes }\end{array}$ \\
\hline
\end{tabular}

behavioral therapies or similar psychosocial treatments should be initiated based on clinical need. The patient's family and friends and other parts of the patient's adaptive system may be engaged to assist in developing a stable footing for the patient. Monitoring for prodromes of episodes should be initiated during the continuation phase. Setting reasonable, attainable, sequential goals for the patient's life is another nonpharmacologic intervention that helps to build toward the maintenance phase.

Despite adequate mood stabilization, some patients continue to exhibit poor social role function. ${ }^{34,35}$ The challenge for clinicians is to provide the level of care required to help the patient make the transition from the continuation phase into the maintenance phase. The best way to prevent relapse is to continue effective treatments. The best way to bring a relapse about is to change or withdraw treatment too early or too quickly.

Maintenance phase. The goals of the maintenance phase are to prevent recurrence and maximize adaptive function. Once patients are functionally stable and can handle "normal" living and working conditions, they are ready to make the transition to maintenance treatment. The first step in the maintenance phase is to continue the pharmacologic interventions that are effective but continually reevaluate their effectiveness. The next step is to begin to gradually taper treatments that are poorly tolerated or presumably nonessential. In some cases, this tapering step may include treatments that were effective for short-term use but carry the risk of toxicity, or have little evidence for continued benefit, if used long-term. Regardless of which treatment is to be tapered, the critical element is that tapering be done gradually so that it can be stopped or the treatment reinstated if early signs of relapse appear.

Interepisode impairment may complicate maintenance treatment. Cognitive impairment, mood instability, and a reduction in or loss of social supports can disrupt the stability achieved to this point. The clinician can combat interepisode impairment by helping patients to strengthen their social adaptive skills and develop a stable social support system.

Dr. Swann reiterated that continuing treatments that showed promise or were necessary during the continuation phase while adding treatments designed to enhance adaptive function, such as insight-oriented treatments, is important to stabilizing the patient. The overall goal during the maintenance phase is to develop and maintain a long-term social and occupational network that can help the patient generate and maintain his or her own stability.

\section{Pharmacotherapy}

Dr. Swann emphasized the importance of identifying pharmacologic treatments that were effective during the acute episode and reviewing the evidence for relapse prevention. Unfortunately, little information about treatments that continue to be effective after the acute episode has resolved is available, and little evidence compares treatments.

Lithium, lamotrigine, and olanzapine have been approved by the FDA for the long-term treatment of bipolar disorder. In addressing bipolar depression, the few placebo-controlled studies of lithium ${ }^{36,37}$ have shown that lithium is effective in patients who have been previously depressed. Other treatments that are supported by placebocontrolled trials for reducing the incidence of depressive relapse in bipolar disorder include divalproex ${ }^{38,39}$ and lamotrigine. ${ }^{40}$ Combination therapy of a mood stabilizer plus olanzapine, ${ }^{41}$ quetiapine, ${ }^{42}$ or risperidone ${ }^{43}$ has also been effective. Although some evidence $^{44,45}$ supports the use of aripiprazole in acute episodes of bipolar mania, no evidence for its long-term use or efficacy in delaying depressive relapse exists. Interestingly, no antidepressants have been shown to prevent depressive relapse in bipolar disorder. For pharmacotherapy, more evidence is available for preventing relapse of mania than preventing relapse of depression in bipolar disorder.

Table 4 summarizes placebocontrolled studies of prevention of depressive episodes. In 2 studies $^{36,37}$ of recently depressed patients, lithium was effective in preventing depressive relapse. In 3 studies ${ }^{37,39,47}$ in which patients were recently manic, lithium was not effective. Divalproex ${ }^{39}$ and lamotrigine $^{47}$ were effective in some of the studies where lithium was not. Bowden et al. ${ }^{39}$ found that lithium did not protect against depression after a manic episode, but divalproex did. The 2 lamotrigine studies ${ }^{40,47}$ found that lamotrigine protected against depressive episodes, regardless of whether patients were recently manic or depressed, while lithium did not. A limi- 


\begin{tabular}{|c|c|c|c|c|c|c|c|}
\hline Reference & $\mathrm{N}$ & Diagnosis & $\begin{array}{l}\text { Duration } \\
(\mathrm{mo})\end{array}$ & $\begin{array}{c}\text { Index } \\
\text { Episode }\end{array}$ & $\begin{array}{c}\text { Result } \\
\text { (Lithium) }\end{array}$ & $\begin{array}{l}\text { Result } \\
\text { (other) }\end{array}$ & Remarks \\
\hline Dunner et $\mathrm{al}^{46}$ & 40 & Bipolar II & 16 & None & - & Not applicable & None \\
\hline \multirow[t]{2}{*}{ Fieve et $\mathrm{al}^{36}$} & 35 & Bipolar I & & Depressed & + & Not applicable & Two thirds of patients had no previous \\
\hline & 18 & Bipolar II & 30 & & & & lithium treatment \\
\hline Prien et $\mathrm{al}^{37}$ & 205 & Bipolar I & 24 & Manic & - & Not applicable & Two thirds of episodes were manic \\
\hline Prien et $\mathrm{al}^{37}$ & 44 & Bipolar II & 24 & Depressed & + & Not applicable & Two thirds of episodes were depressed \\
\hline Bowden et $\mathrm{al}^{39}$ & 372 & Bipolar I & 12 & Manic & - & Divalproex + & $\begin{array}{l}\text { Divalproex also increased response to adjunctive } \\
\text { selective serotonin reuptake inhibitor }\end{array}$ \\
\hline Bowden et $\mathrm{al}^{47}$ & 175 & Bipolar I & 18 & Manic & - & Lamotrigine + & Patients previously stabilized on lamotrigine \\
\hline Calabrese et $\mathrm{al}^{l^{40}}$ & 463 & Bipolar I & 18 & Depressed & - & Lamotrigine + & Patients previously stabilized on lamotrigine \\
\hline
\end{tabular}

tation of these studies, however, is that they were enriched with lamotrigine responders, and so the negative response to lithium may have been exaggerated.

\section{Antidepressants}

Dr. Swann cited a review article by Ghaemi et al. ${ }^{48}$ that compared 7 controlled studies of antidepressant treatment for relapse prevention or maintenance in bipolar disorder. All were negative. No antidepressant has ever been shown to prevent relapse in bipolar disorder in a controlled study. However, antidepressants may still be useful in intermediate- or long-term treatment. Altshuler and colleagues ${ }^{49}$ prospectively compared the effect of antidepressant discontinuation or continuation on depressive relapse risk among patients with bipolar disorder who had been successfully treated with antidepressants during an acute depressive episode. Patients $(\mathrm{N}=84)$ who had achieved remission from a depressive episode with the addition of an antidepressant to an ongoing mood stabilizer were followed for 1 year. Fortythree patients discontinued antidepressant treatment within 6 months after remission and 41 patients continued antidepressants beyond 6 months. $\mathrm{Pa}-$ tients in the discontinuation group experienced a shorter period of euthymia before depressive relapse. One year following antidepressant response, $70 \%$ of the discontinuation group had experienced a depressive relapse compared with $36 \%$ of the continuation group. The risk of depressive relapse was associated with discontinuing antidepressants; speed of relapse was associated with brevity of antidepressant exposure. The authors concluded that antidepressant treatment in combination with a mood stabilizer may be warranted in some patients with bipolar disorder. However, several limitations of this study should be taken into account, including (1) it was neither blinded nor randomized; (2) because it was naturalistic, length of treatment and rate of antidepressant taper varied; (3) reasons for antidepressant discontinuation were not known; and (4) the finding was limited to a subset of $15 \%$ to $20 \%$ of patients who required treatment with an antidepressant and who remained well while taking an antidepressant for more than 2 months. The findings of this study support the model of the continuation phase and the maintenance phase. $\mathrm{Pa}$ tients responsive to antidepressants in the acute phase are likely to tolerate ongoing use in the continuation phase (about 6 to 12 months) and to suffer relapse if it is discontinued during the continuation phase.

Antidepressants can destabilize mood or cause pathologic activation in patients with bipolar disorder. The acute use of antidepressants in bipolar patients may induce mania, mixed states, or rapid cycling, whereas chronic use can lead to loss of response. Concern for the loss of antidepressant response over the course of long-term treatment in patients with bipolar disorder ${ }^{50}$ indicates a need for controlled studies. A recent case se- ries $^{51}$ of subjects with bipolar I disorder who had been taking antidepressants for at least 3 years showed that antidepressants may induce a chronic dysphoric, irritable state and insomnia.

The longer patients are exposed to antidepressant therapy, the more likely they are to relapse into mania or hypomania. Using data from the Stanley Foundation Bipolar Network, Post et al. ${ }^{52}$ reviewed data from 127 patients with bipolar depression who were randomly assigned to 10 weeks of treatment with sertraline, bupropion, or venlafaxine as adjunctive medication to mood stabilizers. Nonresponders were reassigned, and responders were given the option to continue treatment for a year. During acute treatment, switches into hypomania or mania occurred in $9.1 \%$ of patients, and another $9.1 \%$ switched into hypomania alone. During continuation, corresponding switch rates were $16.4 \%$ and $19.2 \%$. Continuation of antidepressants was not associated with a greater rate of switching into mania than discontinuation of antidepressants.

Dr. Swann emphasized that it is important to look for activated depression. Activated depression can be part of the natural history of bipolar disorder; it can be pharmacologic, brought on by prescribed antidepressants, selftreatment, or substance abuse; or it can be environmental, caused by overstimulation or stress. Activated depression is associated with an increased risk for suicide and other health problems, so it is critical that clinicians are alert to its presence. 


\section{Suicide Prevention}

Dr. Swann reemphasized the point made by Dr. Kupfer that depression presents a high risk of premature death in patients with bipolar disorder. Therefore, suicide prevention is an integral part of the long-term treatment of bipolar disorder. Sachs and colleagues ${ }^{53}$ have outlined some strategies that match the strategy to the phase of treatment-acute, continuation, and maintenance. The 3 basic elements are (1) routinely assess the inclination and opportunity for suicide, (2) educate the patient and family members regarding risk factors, and (3) integrate a suicide prevention strategy into a written treatment plan. Basic components of the strategy include the need to:

- Always discuss suicide risk openly.

- Help the patient identify and anticipate prodromes of episodes based on individual history in order to better be proactive about treatment.

- Use medications that reduce recurrence of bipolar episodes and impulsivity.

- Foster a positive forwardlooking approach that includes a social structure, and set sequential, attainable goals.

- Anticipate changes, problems and recurrence, and help the patient to recognize trouble early and to seek solutions in a problem-solving manner.

Most patients can recognize that a depressive or manic episode is coming at least 2 or 3 weeks before the episode occurs. With heightened awareness, it is possible to head off an impending episode. Early prodromes are consistent within the same person but vary from person to person. For example, changes in motivation, the sleep-activity cycle, impulsive behavior (a precursor to mania or mixed states), and interpersonal behavior may be early signs of recurrence of the underlying illness. In the period leading up to a depression, the patient may start hitting the snooze alarm more often, putting things off, avoiding contacts with friends, and reducing the number of activities.

\section{Summary}

Dr. Swann concluded by stating that pharmacologic treatment that is effective for an acute depressive episode of bipolar disorder is likely to be effective as continuation or maintenance treatment. The goals of the continuation phase are prevention of bipolar depression, continued stability, and improvement of adaptive function. During this phase, effective treatments that were essential to producing symptomatic improvement must be continued until the patient is episode-free and has re- turned to the pre-illness level of social occupational functioning along with the previous level of independent living. This period of functional recovery is a high-risk period for suicide.

The goals of the maintenance phase are to prevent recurrence and maximize adaptive function. During this phase, evidence-based, pharmacologic treatment may be continued to maintain mood stability, and treatments that may not be essential for the long term, such as antidepressants and adjunctive treatments, may be carefully, gradually tapered. Integrating pharmacologic and nonpharmacologic treatments and monitoring for suicide risk and activated depression are the most challenging and critical aspects of treating bipolar depression.

\section{Treatment-Resistant Bipolar Depression and Emerging Therapies}

Lauren B. Marangell, M.D., stated that treatment-resistant bipolar disorder is markedly understudied. While numerous medications have been approved by the FDA for the treatment of acute mania, only the combination of olanzapine and fluoxetine has been approved for the acute treatment of bipolar depression (Table 5), and no single-agent pharmacotherapy has been approved for the treatment of bipolar depression or treatment-resistant bipolar disorder. Many clinicians are surprised to learn that the commonly used mood stabilizers lithium, divalproex, and carbamazepine have not been wellstudied in adequately powered randomized trials for acute depression in bipolar disorder and to date appear to be more effective in controlling manic compared to depressive symptoms.

The magnitude of unmet need in treatment-resistant patients with bipolar depression is demonstrated by a recent naturalistic study ${ }^{54}$ of patients with unipolar or bipolar depression who had failed treatment with $\geq 2$ antidepressants in their current depressive episode. This multicenter study tracked outcomes as patients received routine care in the community with any nonexperimental treatment that the treating psychiatrist deemed appropriate, including pharmacotherapies, psychotherapy, and ECT. At the end of 1 year, the remission rates were only $6.7 \%$ based on the Hamilton Rating Scale for Depression (HAM-D) and $3.2 \%$ based on the Inventory of Depressive Symptomatology (IDS). Although this study included patients with unipolar $(\mathrm{N}=109)$ and bipolar disorder $(\mathrm{N}=15)$, remission rates did not differ between the unipolar and bipolar patients. These results may be the best available representation of the long-term course of treatment-resistant depression in bipolar disorder.

\section{Pharmacotherapy}

Given the paucity of data on treatment-resistant patients, Dr. Marangell began by reviewing the available positive randomized, placebo-controlled pharmacotherapy trials for bipolar depression.

Olanzapine and fluoxetine combination. The combination of olanzapine 
Table 5. U.S. Food and Drug Administration-Approved Treatments for

Bipolar Disorder

\begin{tabular}{|llc|}
\hline Mania & Maintenance & Acute Episode of Bipolar Depression \\
\hline Aripiprazole & Aripiprazole & Olanzapine/fluoxetine \\
Carbamazepine & Lamotrigine & \\
Chlorpromazine & Lithium & \\
Divalproex & Olanzapine & \\
Lithium & \\
Olanzapine & \\
Quetiapine & \\
Risperidone & & \\
Ziprasidone & & \\
\hline
\end{tabular}

and fluoxetine is the only FDAapproved treatment for acute bipolar depression. As described by Tohen and colleagues, ${ }^{55}$ the combination of olanzapine and fluoxetine was studied in a randomized, parallel design: 1 group received olanzapine plus fluoxetine in combination $(\mathrm{N}=86), 1$ group received only olanzapine $(\mathrm{N}=370)$, and 1 group received placebo $(\mathrm{N}=377)$. Patient scores on the MADRS dropped 18.5 points from baseline to endpoint at 8 weeks with the olanzapine and fluoxetine combination, which was superior to the modest effect seen with olanzapine alone (15.0 points) or placebo (11.9 points). Nearly half $(48.8 \%)$ of patients in the olanzapine plus fluoxetine group met remission criteria by week 8 compared with $32.8 \%$ in the olanzapine group and $24.5 \%$ in the placebo group. Treatment-emergent mania did not differ among groups, and adverse events for the combination group were similar to those for olanzapine alone.

Quetiapine. Quetiapine is currently the only agent with 2 positive placebocontrolled trials demonstrating efficacy in the acute treatment of bipolar depression. ${ }^{56,57}$ In both trials antidepressant efficacy was noted with both the 300-mg/day and 600-mg/day dosages, with response rates of approximately $58 \%$. Further studies of quetiapine in treatment-resistant patients appear indicated.

Lamotrigine. Lamotrigine has demonstrated efficacy in 1 placebo-controlled trial ${ }^{58}$ in patients with acute bipolar depression, but replication studies have not been positive. Nierenberg and colleagues $^{59}$ recently published one of the only pharmacotherapy studies that evaluated patients with treatmentresistant bipolar depression. In that study, 66 subjects with bipolar I or II disorder and a current major depressive episode nonresponsive to treatment with mood stabilizers plus at least one antidepressant were randomly assigned to adjunctive lamotrigine, inositol, or risperidone for up to 16 weeks. Although there were no significant differences comparing any pair of treatments, the recovery rate with lamotrigine was $23.8 \%$ (95\% $\mathrm{CI}=5.8 \%$ to $41.8 \%$ ), whereas the recovery rate with inositol was $17.4 \%$ (95\% CI $=2.4 \%$ to $32.4 \%$ ) and with risperidone, $4.6 \%(95 \% \mathrm{CI}=0 \%$ to $14.6 \%$ ).

Pramipexole. Two independent groups $^{60,61}$ have reported positive results for adjunctive treatment with the dopamine $\mathrm{D}_{2} / \mathrm{D}_{3}$ receptor agonist pramipexole. Given that both studies included fewer than 30 subjects, further study of these intriguing reports is warranted.

Antidepressants. Dr. Marangell agreed with Dr. Swann that the use of antidepressants in bipolar depression remains controversial. Some clinicians firmly believe that antidepressants are effective treatments for patients with bipolar disorder, although others believe just as firmly that they are ineffective. Anecdotally, many patients in the depressed phase favor a medication called an "antidepressant" as opposed to a medication called a "mood stabilizer." In fact, medications that treat both the manic and depressed phases of the illness or medications that are used in other phases of bipolar disorder may actually be active in alleviating the depression of bipolar disorder as well as antidepressants and produce less lability.

The lability concern with antidepressants in bipolar disorder is that they may actually trigger a manic or hypomanic episode or a rapid-cycling course of illness. Dr. Marangell cited a recently published randomized, controlled trial by Leverich and colleagues $^{62}$ that illustrates this issue. A total of 228 acute (10-week) randomized trials of different antidepressants were conducted among 184 patients who had participated in the former Stanley Foundation Bipolar Network. Of these, 159 patients with bipolar depression were randomly assigned to a mood stabilizer plus antidepressant medication as follows: bupropion $(\mathrm{N}=50)$, sertraline $(\mathrm{N}=50)$, and venlafaxine $(\mathrm{N}=59)$. The acute phase was followed by a continuation phase of up to 1 year. Researchers reported a substantial switch rate into mania of $7.9 \%$ and $14.9 \%$ and hypomania of $11.4 \%$ and $21.8 \%$ in both the acute and continuation phases, respectively. The switch into mania was more likely in patients with bipolar I disorder $(30.8 \%)$ compared with bipolar II disorder (18.6\%). Switches were more common with venlafaxine than sertraline or bupropion. A minority of patients $(23.3 \%)$ had satisfactory clinical responses to antidepressants over time.

Dr. Marangell explained that the variability of responses seen with antidepressants in the context of bipolar depression is one of the reasons that the use of these medications is controversial. Altshuler and colleagues ${ }^{49}$ reported that patients who discontinued antidepressant treatment within the first 6 months after remission had a shorter period of euthymia before depressive relapse. Of the 84 patients with bipolar disorder who achieved remission from a depressive episode with the adjunctive use of an antidepressant, $70 \%$ experienced a depressive relapse within 1 year of antidepressant discontinuation. Although many studies show that antidepressants are ef- 
Figure 3. Remission Rates in 12-Month Study of Vagus Nerve Stimulation in Treatment-Resistant Depression ${ }^{\mathrm{a}}$

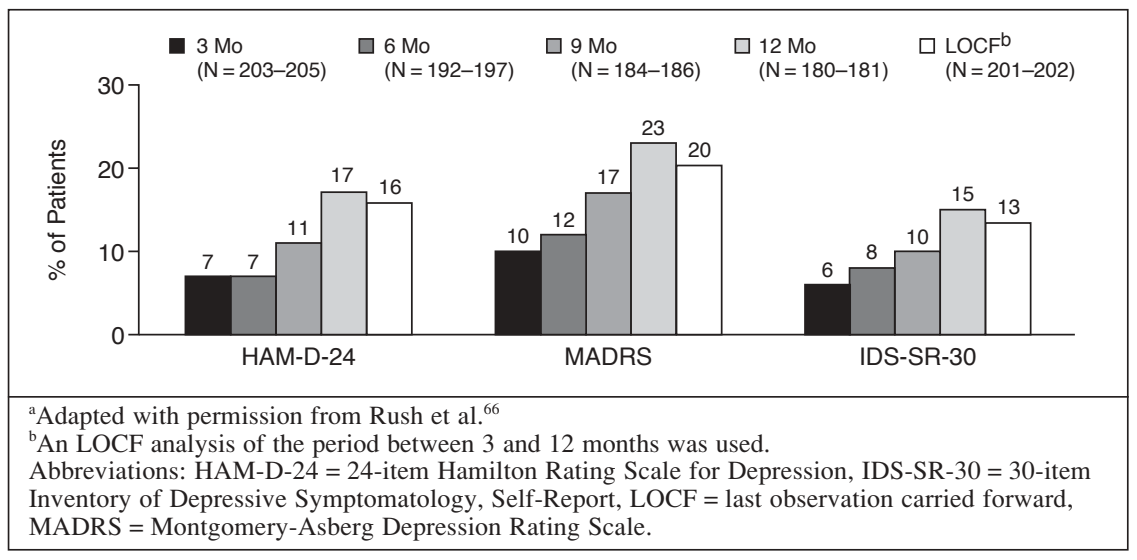

fective, none of these studies involved treatment-resistant groups.

\section{Treatment Devices}

Electroconvulsive therapy. ECT continues to be a highly effective treatment. Most data regarding ECT are for its use in unipolar depression, but clinical evidence shows that ECT also appears to be effective in bipolar depression. ${ }^{63}$ However, response rates and longer-term durability of response are lower in pharmacoresistant patients than in non-treatment-resistant patients. ${ }^{64}$ Dr. Marangell recommended that ECT should be considered as a possible treatment for patients with treatment-resistant bipolar depression, but noted that concerns for long-term management in responsive patients still need to be addressed.

Vagus nerve stimulation. Vagus nerve stimulation (VNS) has received FDA approval as adjunctive treatment for adults with treatment-resistant unipolar or bipolar depression that has failed to respond to 4 or more antidepressant treatments. The primary VNS efficacy study ${ }^{65}$ for treatment-resistant depression was conducted in a mixed population of patients with nonpsychotic major depressive disorder $(\mathrm{N}=199)$ and bipolar depression $(\mathrm{N}=23)$ who were randomly assigned to active VNS treatment $(\mathrm{N}=112)$ or sham treatment $(\mathrm{N}=110)$. At 10 weeks, scores on the 24-item HAM-D indicated a response rate of $15.2 \%$ for the VNS group and $10.0 \%$ for the sham group. Vagus nerve stimulation therapy was well tolerated, but more studies are needed to determine its efficacy for acute treatment.

Long-term response rates for the pivotal trial ${ }^{66}$ showed a consistent pattern of response with each rating scale used to measure improvement - the 24-item HAM-D (HAM-D-24), the MADRS, and the 30-item IDS, SelfReport (IDS-SR-30). Responses accrued with time. In other words, response at 6 months was greater than at 3 months, response at 12 months was greater than at 6 months, and so on. Dr. Marangell pointed out that remission rates are probably the most clinically relevant outcome. As shown in Figure 3, remission rates at the end of 1 year (LOCF) were $15.8 \%$ (32 of 202) as measured by the HAM-D-24, $20.3 \%$ (41 of 202) as measured by the MADRS, and $13.4 \%$ (27 of 201) as measured by the IDS-SR-30. The response and remission rates doubled between 3 and 12 months (LOCF), and changes in the HAM-D-24 and the MADRS scores were statistically significant $(\mathrm{p}<.005)$.

Vagus nerve stimulation is a novel approach to treatment-resistant bipolar disorder and may be considered for patients who have a disease that is characterized by prominent depressive features. Importantly, VNS is considered a long-term treatment and not an acute treatment.

\section{Summary}

In summary, Dr. Marangell reiterated that treatment-resistant bipolar disorder is associated with poor outcomes despite available treatments. The positive controlled data with atypical antipsychotics, especially quetiapine, make this medication an important consideration, although quetiapine has not been studied in treatmentresistant patients. Medication combinations are frequently used, although there is a paucity of controlled data. Some data suggest that lamotrigine may be effective in treatment-resistant depression, but the role of antidepressants remains controversial. Electroconvulsive therapy continues to be used effectively in acute treatmentresistant depression, and VNS offers a longer-term option for those who have not responded to other treatments.

Drug names: aripiprazole (Abilify), bupropion (Wellbutrin and others), carbamazepine (Equetro, Tegretol, and others), chlorpromazine (Thorazine, Sonazine, and others), divalproex (Depakote), lamotrigine (Lamictal), lithium (Eskalith, Lithobid, and others), olanzapine (Zyprexa), olanzapine/ fluoxetine (Symbyax), pramipexole (Mirapex), quetiapine (Seroquel), risperidone (Risperdal), sertraline (Zoloft), venlafaxine (Effexor), ziprasidone (Geodon).

Disclosure of off-label usage: The chair has determined that, to the best of her knowledge, aripiprazole, bupropion, carbamazepine, chlorpromazine, divalproex, inositol, lamotrigine, lithium, olanzapine, pramipexole, quetiapine, risperidone, sertraline, venlafaxine, and ziprasidone are not approved by the U.S. Food and Drug Administration for the treatment of bipolar depression. If you have questions, contact the medical affairs department of the manufacturer for the most recent prescribing information.

\section{References}

1. Calabrese JR, Hirschfeld RMA, Reed M, et al. Impact of bipolar disorder on a US community sample. J Clin Psychiatry 2003; 64:425-432

2. Hirschfeld RM, Lewis L, Vornik LA. Perceptions and impact of bipolar disorder: how far have we really come? results of the National Depressive and Manic-Depressive Association 2000 survey of individuals with bipolar disorder. J Clin Psychiatry 2003;64:161-174

3. Hantouche EG, Akiskal HS, Lancrenon S, et al. Systematic clinical methodology for validating bipolar-II disorder: data in midstream from a French multi-site study (EPIDEP). J Affect Disord 1998;50: $163-173$ 
4. Post RM, Denicoff KD, Leverich GS, et al. Morbidity in 258 bipolar outpatients followed for 1 year with daily prospective ratings on the NIMH life chart method. J Clin Psychiatry 2003;64:680-690

5. Keller MB, Lavori PW, Coryell W, et al. Differential outcome of pure manic, mixed/ cycling, and pure depressive episodes in patients with bipolar illness. JAMA 1986; 255:3138-3142

6. Judd LL, Akiskal HS, Schettler PJ, et al. The long-term natural history of the weekly symptomatic status of bipolar I disorder. Arch Gen Psychiatry 2002;59:530-537

7. Scandinavian Society of Pharmacology Committee of Clinical Investigations (UKU). The UKU Side Effects Rating Scale: scale for the registration of unwanted effects of psychotropics. Acta Psychiatr Scand 1987;76(suppl 334):81-94

8. MacQueen GM, Young LT, Joffe JT. A review of psychosocial outcome in patients with bipolar disorder. Acta Psychiatr Scand 2001;103:163-170

9. Osby U, Brandt L, Correia N, et al. Excess mortality in bipolar and unipolar disorder in Sweden. Arch Gen Psychiatry 2001;58: 844-850

10. Dilsaver SC, Chen Y-W, Swann AC, et al Suicidality, panic disorder and psychosis in bipolar depression, depressive-mania and pure-mania. Psychiatry Res 1997;73: 47-56

11. Kessler RC, McGonagle KA, Zhao S, et al. Lifetime and 12-month prevalence of DSM-III-R psychiatric disorders in the United States: results from the National Comorbidity Survey. Arch Gen Psychiatry 1994;51:8-19

12. Isometsä ET, Henriksson MM, Aro HM, et al. Suicide in bipolar disorder in Finland. Am J Psychiatry 1994;151:1020-1024

13. Krishnan KR. Psychiatric and medical comorbidities of bipolar disorder. Psychosom Med 2005;67:1-8

14. Frank E, Swartz HA, Mallinger AG, et al. Adjunctive psychotherapy for bipolar disorder: effects of changing treatment modality. J Abnorm Psychol 1999;108:579-587

15. Fagiolini A, Kupfer DJ, Houck PR, et al. Obesity as a correlate of outcome in patients with bipolar I disorder. Am J Psychiatry 2003;160:112-117

16. Kupfer DJ. The increasing medical burden in bipolar disorder [commentary]. JAMA 2005;293:2528-2530

17. Frank E, Swartz H, Kupfer D. Interpersonal and social rhythm therapy: managing the chaos of bipolar disorder. Biol Psychiatry 2000;48:593-604

18. Dunner DL, Fieve RR. Clinical factors in lithium carbonate prophylaxis failure. Arch Gen Psychiatry 1974;30:229-233

19. American Psychiatric Association. Diagnostic and Statistical Manual of Mental Disorders, Fourth Edition. Washington, DC: American Psychiatric Association; 1994

20. Schneck CD, Miklowitz DJ, Calabrese JR, et al. Phenomenology of rapid-cycling bipolar disorder: data from the first 500 participants in the Systematic Treatment Enhancement Program. Am J Psychiatry 2004;161:1902-1908

21. Baldessarini RJ, Tondo L, Floris G, et al. Effects of rapid cycling on response to lithium maintenance treatment in 360 bipolar I and II disorder patients. J Affect Disord 2000;61:13-22

22. Kupka RW, Luckenbaugh DA, Post RM, et al. Comparison of rapid-cycling and non-rapid-cycling bipolar disorder based on prospective mood ratings in 539 outpatients. Am J Psychiatry 2005; 162 : 1273-1280

23. Keck PE Jr. Defining and improving response to treatments in patients with bipolar disorder. J Clin Psychiatry 2004;65(suppl 15):25-29

24. Papolos DF, Veit S, Faedda GL, et al. Ultra-ultra rapid cycling bipolar disorder is associated with the low activity catecholamine-O-methyltransferase allele. Mol Psychiatry 1998;3:346-349

25. Green EK, Raybould R, Macgregor S, et al. Genetic variation of brain-derived neurotrophic factor (BDNF) in bipolar disorder: case-control study of over 3000 individuals from the UK. Br J Psychiatry 2006; 188 : 21-25

26. Wehr TA, Goodwin FK. Rapid cycling in manic-depressives induced by tricyclic antidepressants. Arch Gen Psychiatry 1979; 36:555-559

27. Kukopulos A, Reginaldi D, Laddomada P, et al. Course of the manic-depressive cycle and changes caused by treatment. Pharmakopsychiatr Neuropsychopharmakol 1980;13:156-167

28. Calabrese JR, Suppes T, Bowden CL, et al. A double-blind, placebo-controlled, prophylaxis study of lamotrigine in rapidcycling bipolar disorder. J Clin Psychiatry 2000;61:841-850

29. Calabrese JR, Shelton MD, Rapport DJ, et al. A 20-month, double-blind, maintenance trial of lithium versus divalproex in rapidcycling bipolar disorder. Am J Psychiatry 2005;162:2152-2161

30. Bauer MS, Whybrow PD. The effect of changing thyroid function on cyclic affective illness in a human subject. Am J Psychiatry 1986;143:633-636

31. Lish JD, Dime-Meenan S, Whybrow PC, et al. The National Depressive and ManicDepressive Association (DMDA) survey of bipolar members. J Affect Disord 1994; 31:281-294

32. MacQueen GM, Young LT, Robb JC, et al. Effect of number of episodes on wellbeing and functioning of patients with bipolar disorder. Acta Psychiatr Scand 2000;101: 374-381

33. Swann AC. Long-term treatment in bipolar disorder. J Clin Psychiatry 2005;66(suppl 1):7-12

34. Dion GL, Tohen M, Anthony WA, et al. Symptoms and functioning of patients with bipolar disorder six months after hospitalization. Hosp Community Psychiatry 1988; 39:652-657

35. Coryell W, Keller M, Endicott J, et al. Bipolar II illness: course and outcome over a 5-year period. Psychol Med 1989; 19:129-141

36. Fieve RR, Kumbaraci R, Dunner DL. Lithium prophylaxis of depression in bipolar I, bipolar II, and unipolar patients. Am J Psychiatry 1976;133:925-929

37. Prien RF, Klett CJ, Caffey EM. Lithium prophylaxis in recurrent affective illness. Am J Psychiatry 1974;131:198-203

38. Gyulai L, Bowden CL, McElroy SL, et al. Maintenance efficacy of divalproex in the prevention of bipolar depression. Neuropsychopharmacology 2003;28:1374-1382

39. Bowden CL, Calabrese JR, McElroy SL, et al. A randomized, placebo-controlled 12-month trial of divalproex and lithium in treatment of outpatients with bipolar I disorder. Arch Gen Psychiatry 2000;57:481-489

40. Calabrese JR, Bowden CL, Sachs G, et al, for the Lamictal 605 Study Group. A placebo-controlled 18-month trial of lamotrigine and lithium maintenance treatment in recently depressed patients with bipolar I disorder. J Clin Psychiatry 2003;64: 1013-1024

41. Tohen M, Chengappa KNR, Suppes T, et al. Relapse prevention in bipolar I disorder: 18-month comparison of olanzapine plus mood stabiliser $\mathrm{v}$ mood stabiliser alone. Br J Psychiatry 2004;184:337-345

42. Altamura AC, Salvadori D, Madaro D, et al. Efficacy and tolerability of quetiapine in the treatment of bipolar disorder: preliminary evidence from a 12-month open-label study. J Affect Disord 2003;76:267-271

43. Ghaemi SN, Hsu DJ, Rosenquist KJ, et al. Long-term observational comparison of risperidone and olanzapine in bipolar disorder. Ann Clin Psychiatry 2004;16:69-73

44. Keck PE Jr, Marcus R, Tourkodimitris S, et al. A placebo-controlled, double-blind study of the efficacy and safety of aripiprazole in patients with acute bipolar mania. Am J Psychiatry 2003;160:1651-1658

45. Vieta E, Bourin M, Sanchez R, et al. Effectiveness of aripiprazole $\mathrm{v}$ haloperidol in acute bipolar mania: double-blind, randomized, comparative 12-week trial. Br J Psychiatry 2005;187:235-242

46. Dunner DL, Stallone F, Fieve RR, et al. Lithium carbonate and affective disorders: a double-blind study of prophylaxis of depression in bipolar illness. Arch Gen Psychiatry 1976;33:117-120. Correction 1982;39:1344-1345

47. Bowden CL, Calabrese JR, Sachs G, et al. A placebo-controlled 18-month trial of lamotrigine and lithium maintenance treatment in recently manic or hypomanic patients with bipolar I disorder. Arch Gen Psychiatry 2003;60:392-400. Correction 2004:61:680

48. Ghaemi SN, Pardo TB, Hsu DJ. Strategies for preventing the recurrence of bipolar disorder. J Clin Psychiatry 2004;65(suppl 10): $16-23$

49. Altshuler L, Suppes T, Black D, et al. Impact of antidepressant discontinuation after acute bipolar depression remission on rates of depressive relapse at 1-year follow-up. Am J Psychiatry 2003;160:1252-1262

50. Sharma V, Khan M, Smith A. A closer look at treatment resistant depression: is it due to a bipolar diathesis? J Affect Disord 2005; 84:251-257

51. Ei-Mallakh RS, Karippot A. Antidepressant-associated chronic irritable dysphoria (acid) in bipolar disorder: a case series J Affect Disord 2005;84:267-272

52. Post RM, Leverich GS, Nolen WA, et al. A re-evaluation of the role of antidepressants in the treatment of bipolar depression: data from the Stanley Foundation Bipolar Network. Bipolar Disord 2003;5:396-406

53. Sachs GS, Yan LJ, Swann AC, et al. Integration of suicide prevention into outpatient management of bipolar disorder. J Clin Psychiatry 2001;62(suppl 25):3-11 
54. George MS, Rush AJ, Marangell LB, et al. A one-year comparison of vagus nerve stimulation with treatment as usual for treatment-resistant depression. Biol Psychiatry 2005;58:364-373

55. Tohen M, Vieta E, Calabrese J, et al . Efficacy of olanzapine and olanzapinefluoxetine combination in the treatment of bipolar I depression. Arch Gen Psychiatry 2003;60:1079-1088

56. Calabrese JR, Keck PE Jr, Macfadden W, et al. A randomized, double-blind, placebocontrolled trial of quetiapine in the treatment of bipolar I or II depression. Am J Psychiatry 2005;162:1351-1360

57. MacFadden W, Calabrese JR, Ketter TA, et al. Double-blind placebo-controlled, trial of quetiapine in bipolar I and bipolar II depression. Presented at the 159th Annual Meeting of the American Psychiatric Association; May 24, 2006; Toronto, Ontario, Canada

58. Calabrese JR, Bowden CL, Sachs GS, et al, for the Lamictal 602 Study Group. A double-blind placebo-controlled study of lamotrigine monotherapy in outpatients with bipolar I depression. J Clin Psychiatry 1999;60:79-88

59. Nierenberg AA, Ostacher MJ, Calabrese JR, et al. Treatment-resistant bipolar depression: a STEP-BD equipoise randomized effectiveness trial of antidepressant augmentation with lamotrigine, inositol, or risperidone. Am J Psychiatry 2006;163: 210-216

60. Zarate CA Jr, Payne JL, Singh J, et al. Pramipexole for bipolar II depression: a placebo-controlled proof of concept study. Biol Psychiatry 2004;56:54-60

61. Goldberg JF, Burdick KE, Endick CJ. Preliminary randomized, double-blind, placebo-controlled trial of pramipexole added to mood stabilizers for treatmentresistant bipolar depression. Am J Psychiatry 2004;161:564-566

62. Leverich GS, Altshuler LL, Frye MA, et al. Risk of switch in mood polarity to hypomania or mania in patients with bipolar depression during acute and continuation trials of venlafaxine, sertraline, and bupropion as adjuncts to mood stabilizers. Am J Psychiatry 2006;163:232-239

63. Sienaert P, Peuskens J. Electroconvulsive therapy: an effective therapy of medication-resistant bipolar disorder. Bipolar Disord 2006;8:304-306

64. Sackeim HA, Prudic J, Devanand DP, et al. A prospective, randomized, double-blind comparison of bilateral and right unilateral electroconvulsive therapy at different stimulus intensities. Arch Gen Psychiatry 2000;57:425-434

65. Rush AJ, Marangell LB, Sackeim HA, et al. Vagus nerve stimulation for treatment-resistant depression: a randomized, controlled acute phase trial. Biol Psychiatry 2005;58:347-354

66. Rush AJ, Sackeim HA, Marangell LB, et al. Effects of 12 months of vagus nerve stimulation in treatment-resistant depression: a naturalistic study. Biol Psychiatry 2005;58:355-363

For the CME Posttest for this Academic Highlights, see pages 1163-1164. 